\title{
Violence in the Emergency Department: Physicians, Medical Staff, and
}

\section{Patients}

\author{
Nozar Dorestan, ${ }^{1}$ Mehrdad Dargahi-Malamir, ${ }^{2}$ Sara Bahadoram, ${ }^{3}$ and Mohammad Bahadoram ${ }^{4,}$ \\ ${ }^{1}$ Department of Surgery, School of Medicine, Ahvaz Jundishapur University of Medical Sciences, Ahvaz, IR Iran \\ ${ }^{2}$ Department of Internal Medicine, Ahvaz Jundishapur University of Medical Sciences, Ahvaz, IR Iran \\ ${ }^{3}$ Department of Pediatrics, Imam Khomeini Hospital Complex, Tehran University of Medical Sciences, Tehran, IR Iran \\ ${ }^{4}$ Medical Student Research Committee and Social Determinant of Health Research Center, Ahvaz Jundishapur University of Medical Sciences, Ahvaz, IR Iran \\ "Corresponding author: Mohammad Bahadoram, Medical Student Research Committee and Social Determinant of Health Research Center, Ahvaz Jundishapur University of \\ Medical Sciences, Ahvaz, IR Iran. Tel: +98-6133362414, E-mail: mohammadbahadoram@yahoo.com
}

Received 2015 May 06; Revised 2015 October 07; Accepted 2015 November 16.

Keywords: Physicians, Medical Staff, Patients, Emergency Medical Services, Hospitals, Workplace, Violence

\section{Dear Editor,}

Workplace violence behavior causes serious problems in occupational settings (1). The term refers to a context in which people are either verbally or physically abused at the workplace (2). Earlier research suggests that hospital and emergency department (ED) violence is increasing (3). Such violence can involve hospital workers, staff, and even managers in several ways and can be costly to healthcare systems worldwide. In Britain alone, for instance, 30 million pounds are lost annually due to nonattendance caused by violence against medical staff (3). Twenty-two physicians were murdered at work between 1980 and 1989, equal to a quarter of all work-related murders of healthcare staff in that period. Doctors have also been victims of nonfatal violence, verbal abuse, intimidation (4), or even gun threats.

Psychiatrists and ED physicians are exposed to more violence and assaults compared to other medical staff (4), which can be caused by a combination of environmental and clinical factors. Previous research suggests urban locale, the doctors' experience, and patient personality traits such as intoxication, delirium, psychosis, or drug-seeking behavior to be risk factors in psychiatric and ED medicine.

The hospital ED typically has a stressful atmosphere. In such an environment, any encounter has the potential to turn into a violent reaction. Such encounters might be caused by factors such as overcrowded EDs, no or limited access to medical staff, staff carelessness, and long lines, waiting times, and delays. Over-demanding patients and drug abusers who come to the ED for tranquilizers and painkillers might also display violent behavior. For medical staff, this means extra stress, lowered motivation, and a lack of self-efficacy. It can also cause resentment, frustration, dysfunction, absence from work, changing jobs, and resignation (5-9). This is particularly the case for late-shift medical staff, who is constantly worried about their safety (10-12).

Many studies have been conducted on the causes of violence towards physicians and medical staff. However, violence caused in health care centers by the ED physicians themselves remains to be investigated. To prevent such violence, the nature and gravity of the issue must be examined carefully (8), as well as its reasons and implications.

Violent behavior is progressively increasing, and it can have serious financial and social implications for both the victim and the offender. Since various forms of aggression are avoidable, it is best to study the factors that regulate such behavior in order to design efficient approaches to decrease or completely remove it.

Our purpose is to raise awareness and present information regarding the causes and results of the violence instigated by emergency physicians and offer suggestions about how to reduce it. The most mentioned reason for such violence is related to the inappropriate behavior of patients and/or the person accompanying them. The second most frequently mentioned reason was the patients' demand for unwarranted medication or hospital admis$\operatorname{sion}(6,8,9)$.

To minimize such problems, providing patients and visitors with information regarding the ED and treatment procedures can be very helpful. It is also important to manage and limit the visitors' access to the patients' area to reduce the number of visitors and their frequent and demanding requests. To this end, appointing full-time security guards in the ED, as well as a technician-inspector who can direct the visitors, respond to their inquiries, and provide them with the needed information, is suggested. Such a technician-inspector, teamed up with other medical staff, can be trained to pinpoint the potential sources and early signs of aggressive behavior and to respond quickly and ef- 
ficiently to crises. Another suggested solution is to usher the offender to a quiet room to prevent further agitation (9).

The results of surveys showed that another cause of violence on the part of the ED physicians was fatigue. To avoid this problem, it is necessary to provide them with suitable resting quarters and enough time to rest.

Violence is mostly caused by contextual factors external to the individuals themselves (such as poor information or assistance, slow and prolonged admission processes, insufficient waiting rooms, etc.), which can hardly be modified.

To control violence in such a setting, training medical personnel to apply anger management techniques, communicate effectively, and interact and collaborate successfully seems to be the best option. As such, every instance of aggressive behavior should be noted, investigated, and analyzed so as to design prevention policies (9).

ED physicians provide precious services, save many lives, and are valuable assets to society. Reducing jobrelated stress can help improve their performance and the quality of the services they offer.

It is important to note that, although the costs of preventing hospital violence might not be high for a single hospital, these costs increase when considering the overall health system. One of the first steps to take is to educate people so that they realize that certain behavior towards physicians is not acceptable. Such education is a necessary prerequisite for any future intervention.

\section{Footnotes}

Authors' Contribution: Nozar Dorestan, Mohammad Bahadoram, Sara Bahadoram and Mehrdad Dargahi-Malamir reviewed literature and drafted the manuscript.

Funding/Support: This study was financially supported by Ahvaz Jundishapur University of Medical Sciences.

\section{References}

1. Cezar ES, Marziale MH. [Occupational violence problems in an emergency hospital in Londrina, Parana, Brazil]. Cad Saude Publica. 2006;22(1):217-21. [PubMed:16470300].

2. Jones J, Lyneham J. Violence: Part of the job for Australian nurses?. Aust Emerg Nurs J. 2001;4(1):10-4.

3. Whittington $\mathrm{R}$, Wykes $\mathrm{T}$. An evaluation of staff training in psychological techniques for the management of patient aggression.J Clin Nurs. 1996;5(4):257-61. [PubMed: 8718059].

4. Baydin A, Erenler AK. Workplace violence in emergency department and its effects on emergency staff. Int J Emerg Ment Health. 2014;16(2):288-90. [PubMed: 25585480].

5. Presley D, Robinson G. Violence in the emergency department: nurses contend with prevention in the healthcare arena. Nurs Clin North Am. 2002;37(1):161-9. [PubMed: 11818270].

6. Ferns $T$. Violence in the accident and emergency department-an international perspective. Accid Emerg Nurs. 2005;13(3):180-5. doi: 10.1016/j.aaen.2005.03.005. [PubMed:15927470].

7. Edward KL, Ousey K, Warelow P, Lui S. Nursing and aggression in the workplace: a systematic review. Br J Nurs. 2014;23(12):653-4. doi: 10.12968/bjon.2014.23.12.653. [PubMed: 25039630].

8. Kowalenko T, Cunningham R, Sachs CJ, Gore R, Barata IA, Gates D, et al. Workplace violence in emergency medicine: current knowledge and future directions. J Emerg Med. 2012;43(3):523-31. doi: 10.1016/j.jemermed.2012.02.056. [PubMed: 22633755].

9. Laposa JM, Alden LE, Fullerton LM. Work stress and posttraumatic stress disorder in ED nurses/personnel. J Emerg Nurs. 2003;29(1):23-8. [PubMed: 12556825].

10. Marx J, Walls R, Hockberger R. Rosen's Emergency Medicine-Concepts and Clinical Practice. Elsevier Health Sciences; 2013.

11. Tintinalli JE, Krome RL, Ruiz E. Emergency Medicine: A Comprehensive Study Guide. Adv Emerg Nurs J. 1992;14(3):74.

12. Marco CA, Kowalenko T. Competence and challenges of emergency medicine training as reported by emergency medicine residents. $J$ Emerg Med. 2012;43(6):1103-9. doi: 10.1016/j.jemermed.2012.05.033. [PubMed: 22883717]. 